\title{
Neurodevelopmental Underpinnings of Angelman Syndrome
}

\section{Guohui $\mathrm{Li}^{1}$ and Shenfeng Qiu',2*}

${ }^{1}$ Interdisciplinary Graduate Program in Neuroscience, School of Life Science, Arizona State University, USA

${ }^{2}$ Department of Basic Medical Sciences, University of Arizona College of Medicine-Phoenix, Phoenix, AZ 85004, USA

\section{Introduction}

This review briefly discusses key recent research literature on Angelman Syndrome (AS), a rare genetic disorder of neurodevelopmental origin. Dysfunction/inactivation of the maternal $U B E 3 A$ gene and its surrounding chromosome regions has been identified as the causative factor for AS. The human UBE3A gene is located within human chromosome 15q11-13 and encodes an E3 ubiquitin-protein ligase (UBE3A, also called E6 associated protein, E6$\mathrm{AP})$. Due to genetic imprinting of the paternal copy of $U B E 3 A$ gene in many brain regions, loss of function of a single maternal copy of $U B E 3 A$ is highly penetrant and pathogenic. Most of the deficits seen in AS patients have been reproduced in Ube3a gene maternal deficiency mice ('AS mice', $U b e 3 a^{\mathrm{m}-\mathrm{p}+}$ ), thus enabling mechanistic interrogations of AS pathogenesis and therapeutic explorations using mice models. Here we briefly discuss recent advances on AS etiology and identify some challenges in translating mechanistic insights into potential therapeutic interventions. Experimental evidence collected so far indicate impaired maternal $U B E 3 A$ in neurons may contribute to AS deficiency by influencing multifaceted neural developmental processes including cell survival, synaptic transmission, signal transduction, gene expressions.

\section{Genetic Abnormality and Phenotypic Presentation}

Angelman Syndrome (AS) is first reported and named thereafter by pediatrician Harry Angelman in 1965 [1]. AS is a severe debilitating neurodevelopmental disorder characterized by mental retardation, speech impairment, seizures, motor dysfunction, and a high prevalence of autism $[2,3]$. Bone abnormalities, such as brachycephaly, microcephaly, osteoporosis and delayed bone development-associated limb deformity and osteopenia are often co-occurring conditions [4-8]. AS influences the general population with an estimated rate of 1:10000 to 1:40000 in U.S. and the United Kingdom $[9,10]$.

Loss of $U B E 3 A$ gene function was identified as the cause of AS by two research groups in $1997[11,12]$. The human UBE3A gene encodes the E3 uniquitin ligase UBE3A. UBE3A gene is normally expressed in neurons only from the maternally inherited allele, while the paternal allele is silenced by epigenetic mechanisms known as imprinting. Therefore, mutation of the single maternal $U B E 3 A$ allele in neurons leads to near complete loss-of-function of $U B E 3 A$ gene. In the majority of AS patients, UBE3A gene is found inactivated by either intragenic mutation, chromosomal micro deletion in the 15q11-13 regions, paternal uniparental disomy (UPD), or a defective imprinting center (IC) that controls UBE3A transcription [13].

Consistent with this genetic architecture, targeted inactivation of Ube $3 a$ gene in mice [14] also support the role of $U B E 3 A$ protein in AS; upon inheritance of the mutation through the maternal germline, Ube $3 a$ mutant mice (Ube $3 a^{\mathrm{m}-\mathrm{p}+}$, 'AS mice') display salient pathological features of AS. Critical defects in both morphology and function of neurons was found [14]. It is important to note that while deficiency of $U B E 3 A$ causes AS, increased UBE3A gene dosage (e.g. from maternal duplications of the UBE3A-spanning 15q11-q13 region [15] appears to be associated with intellectual and developmental abnormalities seen in autism spectrum disorders, and reproduce most autism features in mouse models $[16,17]$. However, it is currently not clear whether an increase in $U B E 3 A$ dosage alone accounts for the autism phenotypes.

Molecular studies have revealed that knockdown of Ube $3 a$ in mouse increases neuronal death, which might be due to the accruement of $\mathrm{p} 53$ protein, p53-dependent transcription, or deposition of intracellular misfolded polyglutamine proteins [18]. In AS mouse models, the deficiency of Ube 3 a protein causes a reduction of dendrites spine density and dendritic length in multiple brain areas including hippocampus, cortex layer III-V and cerebellum [19,20] AS mice also show defects of dendrite polarization of pyramidal neurons in cortex and hippocampus, decreased dendritic arborization in cortex [21] and decreased synaptic vesicle density in hippocampus [22]. These morphological changes are consistent with the observed functional deficits. For example, decreased miniature excitatory postsynaptic currents (mEPSCs) and synaptic plasticity (such as longterm potentiation (LTP) impairment) are found in AS mouse models [23] which involves down-regulated $\mathrm{N}$-methyl-D-aspartate receptor (NMDAR) function and deficiency of calcium influx. These evidence are indicative that UBE3A is required for normal neuronal activity. On the other hand, cellular UBE3A proteins levels are also affected by neuronal activity. Filonova et al. [24] recently reported that synaptic activation leads to dramatic changes Ube $3 a$ neuronal expression. Both increased neuronal activity by depolarization or fear conditioning behavioral paradigm enhanced neuronal Ube3a levels. The authors also found that in the absence of Ube $3 a$, activity-dependent increase in ERK1/2 phosphorylation was impaired. It may be possible that this altered MAPK pathway may underlie the impaired synaptic plasticity and cognitive function in AS mice.

Intriguingly, experience-dependent maturation of excitatory cortical circuits, and visual cortex function associated with ocular dominance plasticity were found impaired in AS mice, suggesting $U b e 3 a$ is necessary for maintaining developmental cortical plasticity and its loss-of-function may contribute to AS pathophysiology [20]. The same research group also reported that dysfunction of Ube $3 a$ resulted in deficits of fast-spiking inhibitory interneurons in cortex layer II - III and an abnormality of presynaptic vesicle release [25]. Consistent with the role of Ube3a in plasticity, a recent study demonstrated that the

*Corresponding author: Shenfeng Qu, Department of Basic Medical Sciences, University of Arizona College of Medicine Phoenix, Phoenix, AZ 85004, USA, Tel: 602-827-2173; E-mail: sqiu@email.arizona.edu

Received September 15, 2014; Accepted November 10, 2014; Published November 14, 2014

Citation: Li G, Qiu S (2014) Neurodevelopmental Underpinnings of Angelman Syndrome. J Bioanal Biomed 6: 052-056. doi:10.4172/1948-593X.1000111

Copyright: ( $2014 \mathrm{Li} \mathrm{G}$, et al. This is an open-access article distributed under the terms of the Creative Commons Attribution License, which permits unrestricted use, distribution, and reproduction in any medium, provided the original author and source are credited. 
type 5 metabotropic glutamate (mGluR5) receptors-dependent LTD was potentiated in the hippocampus in AS mice [26]. It has been also reported that parvalbumin-positive (PV) interneurons in AS mice are more vulnerable than those of wild type mice in responding to chronic stress. Chronic stress treatment leads to more pronounced decrease of PV neurons in the hippocampus and basolateral amygdala of AS mice, a process that can be antagonized with fluoxetine [27]. AS mice also show abnormality in behavior related to the malfunction of basal ganglia circuits (e.g. instrumental conditioning). These mice have severe difficulty in initial acquisition of lever pressing, and were more habitual and impervious to changes compared with the wild-type control ones. Electrophysiological results revealed that both amplitude and frequency of mEPSCs are decreased in the dorsomedial striatum in AS mice, suggesting specific impairment of synaptic function in an associative corticostriatal circuit [28] that is also shared by the autism spectrum [29].

\section{Pathophysiological and Molecular Changes}

A number of combined genetic and molecular studies have shed light on AS etiology. The UBE3A protein, first identified as the mediator of human papillomavirus types 16 and 18 E6 protein [30,31], regulates ubiquitin-mediated degradation of many proteins, such as the human homologues of yeast Rad23 (HHR23A), which is involved in DNA repair [32], the Src family member Blk [32] and the Rho-GEF pebble (pbl). Many other proteins are regulated by UBE3A when expressed in Drosophila [33]. These proteins include intracellular proteins such as misfolded polyglutamine proteins [18], annexin A1 [34] and the Hsp70/Hsc70 chaperones bound substrates [35] etc.

It has been found that various genetic mechanisms cause the loss (deletion or UPD), inactivation or mutations of maternal UBE3A gene (located in chromosome 15q11-13) [36], (Table 1). Imprinting for $15 \mathrm{q} 11-\mathrm{q} 13$ genes is controlled by a bipartite imprinting center (IC). This IC includes the Angelman syndrome imprinting center (AS-IC) and the Prader-Willi syndrome imprinting center (PWS-IC) [37-39]. Silencing the paternal copy of $U B E 3 A$ gene is likely through paternal expression of a large antisense RNA transcript of $U B E 3 A$ (UBE3A-ATS) and snoRNAs (small nucleolar RNAs) in neurons $[11,12,40]$. It was found that the two types of RNA transcript, sense and antisense, both the products of Ube3a gene, are expressed in a cell-type specific way in the brain. Neurons express maternal sense and paternal antisense, whereas glia express biallelical sense [41]. Furthermore, the disruption of maternal Ube3a gene resulted in an increase of paternal Ube3a-ATS in AS mouse model [42]. The Ube3a-ATS illustrated the inhibitory effect on the expression of paternal UBE3A gene [43], and is consistent with a large scale screening that revealed that maternal biased genes are significantly related to the developing brain [44]. Another study further support that the impaired function of UBE3A in AS patients is related to ubiquitin ligase instead of to its functional coactivator of transcription of the nuclear hormone receptor superfamily, such as the progesterone receptor (PR) [45]. The disturbance of the ubiquitin ligase activity gives rise to the impairment of protein ubiquitination [46] . A recent study showed that the expression level of paternal Ube3a is decreased in mouse neurons after the first postnatal week during which these neurons are undergoing rapid maturation. At the same time, the decrease of paternal Ube $3 a$ was accompanied by the nuclei accumulation of $U b e 3 a$ of maternal origin. Interestingly, in contrast to neuron, glia cells (both astrocyte and oligodendrocyte) seem to express Ube $3 a$ biallelically [47].

The detailed mechanisms on how deficiency of UBE3A leading to
Table 1: Ascertained genetic abnormalities in AS*.

\begin{tabular}{|l|c|}
\hline Genetic abnormality & Percentage in AS cases \\
\hline Maternal deletion of $15 q 11-13$ (De novo) & $\sim 70 \%$ \\
\hline Paternal UPD & $2-5 \%$ \\
\hline Imprinting defects & $2-5 \%$ \\
\hline Mutations/variants of UBE3A gene & $\sim 5-10 \%$ \\
\hline Other causes unidentified & $\sim 10 \%$ \\
\hline
\end{tabular}

Note: According to a report by Ramsden et al. 2010, and also based on the data from the public database Decipher (https://decipher.sanger.ac.uk).

AS are poorly understood. Studies using AS mice have provided some mechanistic insights by demonstrating that Ube3a plays a pivotal role in multiple CNS developmental processes, including cell cycle, signal transduction, transcription and synaptic plasticity [40]. One possible mechanism may be that changes of Ube3a expression can influence the viability of neurons. It was reported that the post mitotic neonatal neurons are decreased after maternal Ube $3 a$ inactivation in AS mouse hippocampus [48]. The loss of neurons may be due to either impaired metabolism or the disturbance of genes involved in cell death process [49], or both. It was also shown that mitochondria in AS mouse exhibited a smaller size in the hippocampus and a partial oxidative phosphorylation defect in the whole brain [22]. Another study revealed that proliferation of neurons was disrupted in AS mice due to the increased expression of cyclin-dependent kinase inhibitor p27, whose degradation is mediated by the Ube3a [50].

Another potential mechanism is that protein synthesis including receptors expression can be affected by Ube $3 a$ dysfunction. A recent study showed that the Golgi apparatus (GA) cistern was swollen and disorganized in Ube3a maternal deficiency mice, and the $\mathrm{pH}$ in GA lumen is increased in cortical neurons. This has implication that a less acidified GA would result in impaired protein sialylation and secretion mechanisms [51]. Previous researches also showed that Ube $3 a$ regulate the degradation and turnover of RhoA-GEF Ephexin-5, activity-regulated cytoskeleton-associated protein (Arc), p53, and p27 via ubiquitination $[13,52,53]$. It has been reported that Arc protein can promote endocytosis of the a-amino-3-hydroxy-5-methyl-4isoxazolepropionic acid-type glutamate receptor (AMPAR), thereby reducing cell surface functional glutamate receptors by facilitating their interaction with dynamin and endophilin [54]. It is not surprising that AMPAR quantity at excitatory synapses was also found decreased, in correlation with an increase of Arc expression after Ube $3 a$ function was disturbed in neurons $[20,55]$.

Another hypothetic cause is that Ube3a abnormality disturbs the regulation of gene expression. A recent study demonstrated that levels of both Ring1B, which ubiquinates nucleosomal histone H2A to regulate gene expression, and histone $\mathrm{H} 2 \mathrm{~A}$, are elevated in many tissues in Ube3a knockout mice [56]. One recent study found that 7 genes are increased and 57 genes are decreased in AS mouse. These genes are functioning in signal transduction, nervous system development and cell death. Some of those genes (Fgf7, Glra1, Mc1r, Nr4a2, Slc5a7 and Epha6) are confirmed of relevant with AS phenotype [49]. It was also shown that elevated Arc level in AS mouse disturbs the brain-derived neurotrophic factor (BDNF) to recruit the postsynaptic density-95 (PSD-95) protein, disrupts association of PSD-95 with TrkB, and the association of PLC $\gamma$ and Grb2-associated binder 1 (Gab1) with TrkB, therefore impairing BDNF, TrkB and PI3K-Akt pathways [57]. Another recent study found that the expression of $\alpha 1$ subunit of sodium/potassium-ATPase ( $\alpha 1-\mathrm{NaKA}$ ) is increased in hippocampus in AS mouse. The abnormal expression likely explains a series of changes such as elevated axon initial segment proteins and altered membrane 
properties including resting potential, threshold potential and action potential. These alterations were corrected by reducing a1-NaKA genetically [58]. This study suggests loss of Ube3a leads to changes in neuronal excitability likely through altered membrane biophysical properties.

\section{Therapeutic Explorations}

The current efforts in therapeutic exploration for AS have been taken on identified putative pathological basis. A conspicuous idea would be to restore the function of the UBE3A in the brain, by retrieval either maternal or paternal copy of the gene. For restoration of maternal Ube $3 a$, one study used recombinant adeno-associated virus (rAAV) to introduce type 2 terminal repeat (TR2) flanked Ube3a into the hippocampus of adult AS mice. The study found that rAAV restored the level of Ube3a in AS hippocampus, rescued the impaired of LTP, and enhanced the cognitive learning as evaluated by Morris Water Maze test [59]. These rescue experiments suggest that neuronal circuit deficits can arise from lack of Ube $3 a$ function per se, and restoration of Ube3a expression could potentially overcome certain aspects of developmental deficits.

The existence of the intact paternal $U B E 3 A$ allele has the intriguing implication that activation of the silent allele may be able to fulfill the functions of the missing maternal ones, in a way analogous to rescuing neural deficits in adult Mecp 2 knockout mice by reinstating the functional Mecp2 gene [60]. In an elegant genetic study, Meng et al. showed that inhibition of Ube3a-ATS expression both in vivo and in vitro could elevate expression of paternal Ube $3 a$ [61]. The activation of paternal Ube3a could be achieved by blocking the paternal Ube3aATS with poly-adenine cassette insertion in AS mouse models. Many resulting AS deficiencies, such as impaired LTP, cognitive deficits, and motor dysfunction were ameliorated [61]. Restoring the paternal Ube $3 a$ expression through non-genetic approaches also seems to hold great promises. Through chemical library screening, Huang et al. (2012) have found that several topoisomerase inhibitors, such as topotecan and irinotecan could resuscitate paternal Ube $3 a$ and rescue cellular function in neurons [62]. Although topoisomerase inhibitors lack specificity on neurons and are likely to be toxic to many tissue types, this study represents a major conceptual breakthrough by showing that rescuing the dysfunctional UBE3A gene in brain can be achieved through bypassing the genetic manipulations.

Maternal Ube3a deficiency in mice is known to impair synaptic transmission and interfere with a critical molecular player in synaptic plasticity, $\mathrm{Ca}^{2+} /$ calmodulin-dependent protein kinase II (CaMKII) $[20,23]$. Weeber et al. showed that AS mice had impaired hippocampal long-term potentiation (LTP) and reduced context-dependent learning, which is correlated with an increased CaMKII phosphorylation at Thr305/Thr306 inhibitory sites and a reduced kinase activity [23]. In a following study [63], the same group further crossed female AS mice with heterozygous males that carried the targeted CaMKIIT305V/T306A mutation, a genetic manipulation that prevents inhibitory phosphorylation of CaMKII and elevates CaMKII activity. Intriguingly, a reduction of CaMKII inhibitory phosphorylation was able to rescue the motor deficits, seizures, LTP impairment and the hippocampus dependent learning. Collectively, these findings indicate misregulation of CaMKII may be a molecular substrate underlying the neurobehavioral deficits in AS. The notion that restoring affected signal transduction pathways may alleviate AS pathology is also supported by another recent study [57]. Cao et al. reported that altered LTP in AS mouse model can be corrected after the TrkB signal pathway was restored by using a bridged cyclic peptide (CN2097) to interfere the interaction between the increased Arc and PSD-95.

\section{Outstanding Questions and Major Challenges}

Despite these emerging successes in restore neural functions in AS mouse models, outstanding questions and challenges remain in the field. For example,

1. What is the definite role of UBE3A in neural connections or circuits within and between many brain regions, and in what molecular context is UBE3A involved to regulate synaptic development, transmission, and plasticity? Why increased UBE3A dosage is more represented in autism spectrum disorders $[16,17]$ ?

2. How does UBE3A differentially affect both excitatory and inhibitory synapses, favoring an enhanced local circuit hyperexcitability $[20,25]$ ?

3. The molecular mechanisms by which $U B E 3 A$ deficiency lead to AS remain enigmatic. The protein substrates of UBE3A in neurons remain to be identified [10]. Arc, Sacsin, HHR23A and Ephexin 5 represent only a small number of proteins known to be directly regulated by UBE3A in neurons. Revealing more neuronal molecular substrates or interactomes and how deficiency of maternal UBE3A disrupts cellular homeostasis can be illuminating for AS pathogenesis and molecular intervations.

4. The dramatic variations of symptoms among AS patients imply the contributions of other elusive and perhaps much more complex causes other than maternal UBE3A dysfunction. For example, other genes such as $\mathrm{GABA}_{\mathrm{A}}$ receptor $\beta 3$ subunit $(G A B R B 3)$ gene which locates within the chromosome 15q11-13 locus have been proved playing a role in the AS development. The impaired expression of $G A B R B 3$ can render featured phenotypes of AS in mice. These results raised the questions of the definite role of the GABRB3 and its relation to $U B E 3 A$ in AS genesis [64].

5. Some discrepancies exist between AS model mouse behavior and AS patient clinical features. AS mice showed normal social seeking and activity level in contrast to the frequently observed behavioral deficits of AS patients [65]. This may be explained by the larger size of genetic defect in patients than that of the AS mice. Further observations on the variations in eating behavior and body growth among patients with different genetic deficits, specifically patients with big deletion or ones with UPD implied that other factors within the 15q11-13 locus may play a role in the pathogenesis too $[36,66]$.

6. On the forefront of AS therapeutic endeavors, the potential of topoisomerase inhibitors in restoring UBE3A expression and correcting AS pathophysiology awaits further experimental validation and extrapolation.

\section{References}

1. Angelman H (1965) Puppet Children: A report of three cases. Developmental medicine and child neurology 7: 681-688.

2. Williams CA, Driscoll DJ, Dagli Al (2010) Clinical and genetic aspects of Angelman syndrome. Genet Med 12: 385-395.

3. Bird LM (2014) Angelman syndrome: review of clinical and molecular aspects Appl Clin Genet 7: 93-104.

4. Oiglane-Shlik E, Rein R, Tillmann V, Talvik T, Ounap K (2005) A female with Angelman syndrome and unusual limb deformities. Pediatr Neurol 33: 66-69.

5. Williams CA, Beaudet AL, Clayton-Smith J, Knoll JH, Kyllerman M, et al. (2006) Angelman syndrome 2005: updated consensus for diagnostic criteria. Am J Med Genet A 140: 413-418. 
Citation: Li G, Qiu S (2014) Neurodevelopmental Underpinnings of Angelman Syndrome. J Bioanal Biomed 6: 052-056. doi:10.4172/1948593X.1000111

6. Coppola G, Verrotti A, Mainolfi C, Auricchio G, Fortunato D, et al. (2007) Bone mineral density in angelman syndrome. Pediatr Neurol 37: 411-416.

7. Kyllerman M (2013) Angelman syndrome. Handb Clin Neurol 111: 287-290.

8. Thibert RL, Larson AM, Hsieh DT, Raby AR, Thiele EA (2013) Neurologic manifestations of Angelman syndrome. Pediatr Neurol 48: 271-279.

9. Buckley RH, Dinno N, Weber P (1998) Angelman syndrome: are the estimates too low? Am J Med Genet 80: 385-390.

10. Clayton-Smith J, Laan $L$ (2003) Angelman syndrome: a review of the clinical and genetic aspects. J Med Genet 40: 87-95.

11. Kishino T, Lalande M, Wagstaff J (1997) UBE3A/E6-AP mutations cause Angelman syndrome. Nat Genet 15: 70-73.

12. Matsuura T, Sutcliffe JS, Fang P, Galjaard RJ, Jiang YH, et al. (1997) De novo truncating mutations in E6-AP ubiquitin-protein ligase gene (UBE3A) in Angelman syndrome. Nat Genet 15: 74-77.

13. Mabb AM, Judson MC, Zylka MJ, Philpot BD (2011) Angelman syndrome: insights into genomic imprinting and neurodevelopmental phenotypes. Trends Neurosci 34: 293-303.

14. Jiang YH, Armstrong D, Albrecht U, Atkins CM, Noebels JL, et al. (1998) Mutation of the Angelman ubiquitin ligase in mice causes increased cytoplasmic p53 and deficits of contextual learning and long-term potentiation. Neuron 21: 799-811.

15. Cook EH Jr, Lindgren V, Leventhal BL, Courchesne R, Lincoln A, et al. (1997) Autism or atypical autism in maternally but not paternally derived proximal $15 q$ duplication. Am J Hum Genet 60: 928-934.

16. Nakatani J, Tamada K, Hatanaka F, Ise S, Ohta H, et al. (2009) Abnormal behavior in a chromosome-engineered mouse model for human 15q11-13 duplication seen in autism. Cell 137: 1235-1246.

17. Smith SE, Zhou YD, Zhang G, Jin Z, Stoppel DC, et al. (2011) Increased gene dosage of Ube3a results in autism traits and decreased glutamate synaptic transmission in mice. Sci Transl Med 3: 103ra97.

18. Mishra A, Dikshit P, Purkayastha S, Sharma J, Nukina N, et al. (2008) E6-AP promotes misfolded polyglutamine proteins for proteasomal degradation and suppresses polyglutamine protein aggregation and toxicity. J Biol Chem 283 : 7648-7656.

19. Dindot SV, Antalffy BA, Bhattacharjee MB, Beaudet AL (2008) The Angelman syndrome ubiquitin ligase localizes to the synapse and nucleus, and maternal deficiency results in abnormal dendritic spine morphology. Hum Mol Genet 17: 111-118.

20. Yashiro K, Riday TT, Condon KH, Roberts AC, Bernardo DR, et al. (2009) Ube $3 a$ is required for experience-dependent maturation of the neocortex. Nat Neurosci 12: 777-783.

21. Miao S, Chen R, Ye J, Tan GH, Li S, et al. (2013) The Angelman syndrome protein Ube $3 a$ is required for polarized dendrite morphogenesis in pyramidal neurons. J Neurosci 33: 327-333.

22. Su H, Fan W, Coskun PE, Vesa J, Gold JA, et al. (2011) Mitochondrial dysfunction in CA1 hippocampal neurons of the UBE3A deficient mouse model for Angelman syndrome. Neurosci Lett 487: 129-133.

23. Weeber EJ, Jiang YH, Elgersma Y, Varga AW, Carrasquillo Y, et al. (2003) Derangements of hippocampal calcium/calmodulin-dependent protein kinase II in a mouse model for Angelman mental retardation syndrome. Journal of neuroscience 23: 2634-2644.

24. Filonova I, Trotter JH, Banko JL, Weeber EJ (2014) Activity-dependen changes in MAPK activation in the Angelman Syndrome mouse model. Learn Mem 21: 98-104

25. Wallace ML, Burette AC, Weinberg RJ, Philpot BD (2012) Maternal loss of Ube3a produces an excitatory/inhibitory imbalance through neuron typespecific synaptic defects. Neuron 74: 793-800.

26. Pignatelli M, Piccinin S, Molinaro G, Di Menna L, Riozzi B, et al. (2014) Changes in mGlu5 receptor-dependent synaptic plasticity and coupling to homer proteins in the hippocampus of Ube $3 \mathrm{~A}$ hemizygous mice modeling angelman syndrome. Journal of neuroscience 34: 4558-4566.

27. Godavarthi SK, Sharma A, Jana NR (2014) Reversal of reduced parvalbumin neurons in hippocampus and amygdala of Angelman syndrome model mice by chronic treatment of fluoxetine. J Neurochem 130: 444-454.

28. Hayrapetyan V, Castro S, Sukharnikova T, Yu C, Cao X, et al. (2013) Regionspecific impairments in striatal synaptic transmission and impaired instrumental learning in a mouse model of Angelman syndrome. The European journal of neuroscience 39: 1018-1025

29. Rothwell PE, Fuccillo MV, Maxeiner S, Hayton SJ, Gokce O, et al. (2014) Autism-associated neuroligin-3 mutations commonly impair striatal circuits to boost repetitive behaviors. Cell 158: 198-212.

30. Huibregtse JM, Scheffner M, Howley PM (1991) A cellular protein mediates association of p53 with the E6 oncoprotein of human papillomavirus types 16 or 18. EMBO J 10: 4129-4135.

31. Huibregtse JM, Scheffner M, Howley PM (1993) Cloning and expression of the cDNA for E6-AP, a protein that mediates the interaction of the human papillomavirus E6 oncoprotein with p53. Mol Cell Biol 13: 775-784.

32. Oda H, Kumar S, Howley PM (1999) Regulation of the Src family tyrosine kinase Blk through E6AP-mediated ubiquitination. Proc Natl Acad Sci U S A 96: 9557-9562.

33. Reiter LT, Seagroves TN, Bowers M, Bier E (2006) Expression of the Rho-GEF $\mathrm{Pbl} / \mathrm{ECT} 2$ is regulated by the UBE3A E3 ubiquitin ligase. Hum Mol Genet 15 2825-2835.

34. Shimoji T, Murakami K, Sugiyama Y, Matsuda M, Inubushi S, et al. (2009) Identification of annexin $\mathrm{A} 1$ as a novel substrate for E6AP-mediated ubiquitylation. J Cell Biochem 106: 1123-1135.

35. Mishra A, Godavarthi SK, Maheshwari M, Goswami A, Jana NR (2009b) The ubiquitin ligase E6-AP is induced and recruited to aggresomes in response to proteasome inhibition and may be involved in the ubiquitination of Hsp70 bound misfolded proteins. Journal of biological chemistry 284: 10537-10545.

36. Lossie AC, Whitney MM, Amidon D, Dong HJ, Chen P, et al. (2001) Distinct phenotypes distinguish the molecular classes of Angelman syndrome. J Med Genet 38: 834-845.

37. Nicholls RD, Saitoh S, Horsthemke B (1998) Imprinting in Prader-Willi and Angelman syndromes. Trends Genet 14: 194-200.

38. Chamberlain SJ, Brannan CI (2001) The Prader-Willi syndrome imprinting center activates the paternally expressed murine Ube3a antisense transcript but represses paternal Ube3a. Genomics 73: 316-322.

39. Ramsden SC, Clayton-Smith J, Birch R, Buiting K (2010) Practice guidelines for the molecular analysis of Prader-Willi and Angelman syndromes. BMC Med Genet 11: 70.

40. Girardot M, Cavaillé J, Feil R (2012) Small regulatory RNAs controlled by genomic imprinting and their contribution to human disease. Epigenetics 7 : 1341-1348.

41. Yamasaki $\mathrm{K}$, Joh $\mathrm{K}$, Ohta $\mathrm{T}$, Masuzaki $\mathrm{H}$, Ishimaru $\mathrm{T}$, et al. (2003) Neurons but not glial cells show reciprocal imprinting of sense and antisense transcripts of Ube3a. Hum Mol Genet 12: 837-847.

42. Landers M, Calciano MA, Colosi D, Glatt-Deeley H, Wagstaff J, et al. (2005) Maternal disruption of Ube3a leads to increased expression of Ube3a-ATS in trans. Nucleic Acids Res 33: 3976-3984.

43. Meng L, Person RE, Beaudet AL (2012) Ube3a-ATS is an atypical RNA polymerase II transcript that represses the paternal expression of Ube3a. Hum Mol Genet 21: 3001-3012.

44. Gregg C, Zhang J, Weissbourd B, Luo S, Schroth GP, et al. (2010) Highresolution analysis of parent-of-origin allelic expression in the mouse brain. Science 329: 643-648

45. Nawaz Z, Lonard DM, Smith CL, Lev-Lehman E, Tsai SY, et al. (1999) The Angelman syndrome-associated protein, E6-AP, is a coactivator for the nuclea hormone receptor superfamily. Molecular and cellular biology 19: 1182-1189.

46. Cooper EM, Hudson AW, Amos J, Wagstaff J, Howley PM (2004) Biochemical analysis of Angelman syndrome-associated mutations in the E3 ubiquitin ligase E6-associated protein. J Biol Chem 279: 41208-41217.

47. Judson MC, Sosa-Pagan JO, Del Cid WA, Han JE, Philpot BD (2014) Allelic specificity of Ube3a expression in the mouse brain during postnatal development. J Comp Neurol 522: 1874-1896. 
Citation: Li G, Quu S (2014) Neurodevelopmental Underpinnings of Angelman Syndrome. J Bioanal Biomed 6: 052-056. doi:10.4172/1948593X.1000111

48. Mardirossian S, Rampon C, Salvert D, Fort P, Sarda N (2009) Impaired hippocampal plasticity and altered neurogenesis in adult Ube3a maternal deficient mouse model for Angelman syndrome. Exp Neurol 220: 341-348.

49. Low D, Chen KS (2010) Genome-wide gene expression profiling of the Angelman syndrome mice with Ube3a mutation. Eur J Hum Genet 18: 12281235.

50. Mishra A, Godavarthi SK, Jana NR (2009) UBE3A/E6-AP regulates cell proliferation by promoting proteasomal degradation of p27. Neurobiol Dis 36: 26-34.

51. Condon KH, Ho J, Robinson CG, Hanus C, Ehlers MD (2013) The Angelman syndrome protein Ube3a/E6AP is required for Golgi acidification and surface protein sialylation. J Neurosci 33: 3799-3814.

52. Margolis SS, Salogiannis J, Lipton DM, Mandel-Brehm C, Wills ZP, et al. (2010) EphB-mediated degradation of the RhoA GEF Ephexin5 relieves a developmental brake on excitatory synapse formation. Cell 143: 442-455.

53. Kühnle S, Mothes B, Matentzoglu K, Scheffner M (2013) Role of the ubiquitin ligase E6AP/UBE3A in controlling levels of the synaptic protein Arc. Proc Nat Acad Sci U S A 110: 8888-8893.

54. Chowdhury S, Shepherd JD, Okuno H, Lyford G, Petralia RS, et al. (2006) Arc/Arg3.1 interacts with the endocytic machinery to regulate AMPA receptor trafficking. Neuron 52: 445-459.

55. Greer PL, Hanayama R, Bloodgood BL, Mardinly AR, Lipton DM, et al. (2010) The Angelman Syndrome protein Ube3A regulates synapse development by ubiquitinating arc. Cell 140: 704-716.

56. Zaaroor-Regev D, de Bie P, Scheffner M, Noy T, Shemer R, et al. (2010) Regulation of the polycomb protein Ring1B by self-ubiquitination or by E6-AP may have implications to the pathogenesis of Angelman syndrome. Proc Natl Acad Sci USA 107: 6788-6793.

57. Cao C, Rioult-Pedotti MS, Migani P, Yu CJ, Tiwari R, et al. (2013) Impairment of TrkB-PSD-95 signaling in Angelman syndrome. PLoS Biol 11: e1001478.
58. Kaphzan H, Buffington SA, Ramaraj $A B$, Lingrel JB, Rasband $M N$, et al (2013) Genetic reduction of the $\hat{I} \pm 1$ subunit of $\mathrm{Na} / \mathrm{K}-\mathrm{ATP}$ ase corrects multiple hippocampal phenotypes in Angelman syndrome. Cell Rep 4: 405-412.

59. Daily JL, Nash K, Jinwal U, Golde T, Rogers J, et al. (2011) Adeno-associated virus-mediated rescue of the cognitive defects in a mouse model for Angelman syndrome. PLoS One 6: e27221.

60. Guy J, Gan J, Selfridge J, Cobb S, Bird A (2007) Reversal of neurological defects in a mouse model of Rett syndrome. Science 315: 1143-1147.

61. Meng L, Person RE, Huang W, Zhu PJ, Costa-Mattioli M, et al. (2013) Truncation of Ube3a-ATS unsilences paternal Ube3a and ameliorates behavioral defects in the Angelman syndrome mouse model. PLoS Genet 9: e1004039.

62. Huang HS, Allen JA, Mabb AM, King IF, Miriyala J, et al. (2011) Topoisomerase inhibitors unsilence the dormant allele of Ube3a in neurons. Nature 481: 185189

63. van Woerden GM, Harris KD, Hojjati MR, Gustin RM, Qiu S, et al. (2007) Rescue of neurological deficits in a mouse model for Angelman syndrome by reduction of alphaCaMKII inhibitory phosphorylation. Nature neuroscience 10: 280-282.

64. DeLorey TM, Handforth A, Anagnostaras SG, Homanics GE, Minassian BA et al. (1998) Mice lacking the beta3 subunit of the GABAA receptor have the epilepsy phenotype and many of the behavioral characteristics of Angelman syndrome. Journal of neuroscience: the official journal of the Society for Neuroscience 18: 8505-8514.

65. Allensworth M, Saha A, Reiter LT, Heck DH (2011) Normal social seeking behavior, hypoactivity and reduced exploratory range in a mouse model of Angelman syndrome. BMC Genet 12: 7

66. Mertz LG, Christensen R, Vogel I, Hertz JM Østergaard JR (2014) Eating behavior, prenatal and postnatal growth in Angelman syndrome. Res Dev Disabil 35: 2681-2690. 